\title{
REGULARITY AND BERNSTEIN-TYPE RESULTS FOR NONLOCAL MINIMAL SURFACES
}

\author{
ALESSIO FIGALLI AND ENRICO VALDINOCI
}

\begin{abstract}
We prove that, in every dimension, Lipschitz nonlocal minimal surfaces are smooth. Also, we extend to the nonlocal setting a famous theorem of De Giorgi [5] stating that the validity of Bernstein's theorem in dimension $n+1$ is a consequence of the nonexistence of $n$-dimensional singular minimal cones in $\mathbb{R}^{n}$.
\end{abstract}

\section{INTRODUCTION}

Given $n \geqslant 1$ and $s \in(0,1)$, we investigate the regularity of nonlocal $s$-minimal surfaces in $\mathbb{R}^{n+1}$. To begin, we recall the notion of $s$-perimeter and $s$-minimal surface, as introduced in [3].

Given two disjoint measurable sets $F, G \subseteq \mathbb{R}^{n+1}$ we consider the $s$-interaction between them defined by

$$
L(F, G):=\iint_{F \times G} \frac{d x d y}{|X-Y|^{n+1+s}} .
$$

Given a measurable set $E$ and a bounded set $\Omega \subset \mathbb{R}^{n+1}$, the " $s$-perimeter" of the set $E$ inside $\Omega$ is defined as

$$
\operatorname{Per}_{s}(E, \Omega):=L\left(E \cap \Omega, \mathbb{R}^{n+1}\right)+L(E \backslash \Omega, \Omega \backslash E) .
$$

We say that $E$ is a " $s$-minimal surface" in $\Omega$ if $\operatorname{Per}_{s}(E, \Omega)<+\infty$ and for any measurable set $F \subseteq \mathbb{R}^{n+1}$ with $F \backslash \Omega=E \backslash \Omega$ we have that

$$
\operatorname{Per}_{s}(E, \Omega) \leqslant \operatorname{Per}_{s}(F, \Omega) .
$$

If $E$ is a $s$-minimal surface in any ball, we simply say that $E$ is a $s$-minimal surface. Namely, $s$-minimal surfaces are local minimizers of the $s$-perimeter functional. The " $s$-mean curvature" of $E$ at a point $X \in \partial E$ is defined by

$$
I[E](X):=\int_{\mathbb{R}^{n+1}} \frac{\chi_{E}(Y)-\chi_{E^{c}}(Y)}{|X-Y|^{n+1+s}} d y,
$$

where $E^{c}:=\mathbb{R}^{n+1} \backslash E$. We remark that if $\partial E$ is $C^{2}$ in a neighborhood of $X$, then $I[E](X)$ is well-defined in the principal value sense. On the other hand, while a priori a $s$-minimal surface $E$ may not be smooth, it is shown in [3] that it satisfies the equation $I[E](X)=0$ for any $X \in \partial E$ in a suitable viscosity sense (in particular, it satisfies the equation in the classical sense at every point where $\partial E$ is $C^{2}$ ).

With this notation, s-minimal surfaces have vanishing $s$-mean curvature, and the analogy with the classical perimeter case is evident. To make the analogy even stronger, we recall that, as $s \nearrow 1$, the $s$-perimeter converges to the classical perimeter, with good geometric and functional analytic properties, see $[1,4]$.

From the results in $[2,3,6]$ it is known that boundaries of $s$-minimal surfaces are $C^{\infty}$ with the possible exception of a closed singular set of Hausdorff dimension at most $n-3$.

2010 Mathematics Subject Classification. 49Q05, 35B65, 35R11, 28A75.

Key words and phrases. s-minimal surfaces, regularity theory, Bernstein's Theorem.

Supported by NSF Grant DMS-1262411 (AF) and ERC Grant $\varepsilon 277749$ (EV). 
The first result of this paper shows that Lipschitz $s$-minimal surfaces are smooth. Notice that, in the classical case, this result is a consequence of the De Giorgi-Nash Theorem on the Hölder regularity of solutions to uniformly elliptic equations in divergence form. However, in this nonlocal setting it does not seem possible to use the regularity theory for nonlocal equations to deduce this result and we need to employ geometric arguments instead.

Theorem 1.1. Let $n \geqslant 1$ and $E$ be a s-minimal surface in $B_{1} \subset \mathbb{R}^{n+1}$. Suppose that $\partial E \cap B_{1}$ is locally Lipschitz. Then $\partial E \cap B_{1}$ is $C^{\infty}$.

We say that a $s$-minimal surface $E$ is a "s-minimal graph" if it can be written as a global graph in some direction (that is, up to a rotation, $E=\left\{(x, \tau) \in \mathbb{R}^{n} \times \mathbb{R}: \tau<u(x)\right\}$ for some function $u: \mathbb{R}^{n} \rightarrow \mathbb{R}$ ), and it is a "s-minimal cone" if it is a cone (that is, up to a translation, $E=t E$ for any $t>0$ ). A variant of the techniques used in the proof of Theorem 1.1 allows us to show that the validity of Bernstein's theorem in dimension $n+1$ is a consequence of the nonexistence of $n$-dimensional singular $s$-minimal cones in $\mathbb{R}^{n}$, thus extending to the fractional case a famous result of De Giorgi for minimal surfaces [5]:

Theorem 1.2. Let $E=\left\{(x, \tau) \in \mathbb{R}^{n} \times \mathbb{R}: \tau<u(x)\right\}$ be a s-minimal graph, and assume there there are no singular s-minimal cones in dimension $n$ (that is, if $\mathscr{C} \subset \mathbb{R}^{n}$ is a s-minimal cone, then $\mathscr{C}$ is a half-space). Then $u$ is an affine function (thus $E$ is a half-space).

The above result combined with the non-existence of $s$-minimal cones in dimension $n \leqslant 2$ (see [6]) implies the following:

Corollary 1.3. Let $E=\left\{(x, \tau) \in \mathbb{R}^{n} \times \mathbb{R}: \tau<u(x)\right\}$ be a s-minimal graph, and assume that $n \in\{1,2\}$. Then $u$ is an affine function.

When $n=1$ Corollary 1.3 is a particular case of the result in [6], but for $n=2$ the result is new.

The paper is organized as follows. Some preliminary results on Lipschitz functions are collected in Section 2, and a useful observation on the asymptotic behavior of the $s$-minimal cones at large scale is given in Section 3. Then, the proofs of Theorems 1.1 and 1.2 are given in Sections 4 and 5, respectively.

\section{Technical Lemmata on Lipschitz functions}

This section contains some auxiliary results of elementary nature.

In the first lemma we show that Lipschitz functions whose gradient is almost constant in a suitably large set need to be uniformly close to an affine hyperplane:

Lemma 2.1. Let $M>0$ and $\omega \in \mathbb{R}^{n}$ with $|\omega| \leqslant M$. Given $\varepsilon>0$ there exists $\delta=\delta(n, \varepsilon, M)>0$, such that the following holds: if $u: B_{1} \rightarrow \mathbb{R}$ is a $M$-Lipschitz function satisfying

$$
\left|\left\{x \in B_{1}:|\nabla u(x)-\omega| \geqslant \delta\right\}\right| \leqslant \delta
$$

then $|u(x)-u(0)-\omega \cdot x| \leqslant \varepsilon$ for any $x \in B_{1}$.

Proof. In this proof $C$ will denote a generic constant depending only $M$, which may change from line to line. Set $w(x):=u(x)-\omega \cdot x$. It is immediate to check that $w$ is $(2 M)$-Lipschitz and from our assumptions on $u$ we get

$$
\int_{B_{1}}|\nabla w(x)| d x=\int_{B_{1} \cap\{|\nabla w|<\delta\}}|\nabla w(x)| d x+\int_{B_{1} \cap\{|\nabla w| \in[\delta, 2 M]\}}|\nabla w(x)| d x \leqslant C \delta .
$$

Hence, by Hölder inequality,

$$
\|\nabla w\|_{L^{n+1}\left(B_{1}\right)} \leqslant\|\nabla w\|_{L^{1}\left(B_{1}\right)}^{1 /(n+1)}\|\nabla w\|_{L^{\infty}\left(B_{1}\right)}^{n /(n+1)} \leqslant C \delta^{1 /(n+1)}
$$


and applying Sobolev inequality in $W^{1, n+1}\left(B_{1}\right)$ we deduce that there exists a constant $\ell \in \mathbb{R}$ such that

Since

$$
\|w-\ell\|_{L^{\infty}\left(B_{1}\right)} \leqslant C\|\nabla w\|_{L^{n+1}\left(B_{1}\right)} \leqslant C \delta^{1 /(n+1)} .
$$

$$
\|w-w(0)\|_{L^{\infty}\left(B_{1}\right)} \leqslant\|w-\ell\|_{L^{\infty}\left(B_{1}\right)}+|\ell-w(0)| \leqslant 2\|w-\ell\|_{L^{\infty}\left(B_{1}\right)},
$$

this concludes the proof with $\varepsilon=C \delta^{1 /(n+1)}$.

In the next result we observe that if a Lipschitz function has local growth close to the maximal one at many points, then it needs to be uniformly close to an affine map:

Proposition 2.2. Let $M>0$. Then, for any $\varepsilon>0$ there exists $\mu=\mu(n, \varepsilon, M) \in(0, M)$ such that the following holds: fix $\sigma \in \partial B_{1}$, and let $u: B_{1} \rightarrow \mathbb{R}$ be a M-Lipschitz function satisfying

$$
\left|\left\{x \in B_{1}: u\left(x+t_{k} \sigma\right)-u(x) \geqslant(M-\mu) t_{k}\right\}\right| \geqslant(1-\mu)\left|B_{1}\right|
$$

for some sequence $t_{k} \searrow 0$. Then $|u(x)-u(0)-M \sigma \cdot x| \leqslant \varepsilon$ for all $x \in B_{1}$.

Proof. Up to a rotation we can assume that $\sigma=e_{1}$. Set

$$
A_{k}:=\left\{x \in B_{1}: u\left(x+t_{k} e_{1}\right)-u(x) \geqslant(M-\mu) t_{k}\right\}
$$

and

$$
A_{\star}:=\bigcap_{k=0}^{+\infty} \bigcup_{j=k}^{+\infty} A_{j}
$$

Notice that $\left|A_{k}\right| \geqslant(1-\mu)\left|B_{1}\right|$ (thanks to (2.2) ) and

$$
\bigcap_{k=0}^{m} \bigcup_{j=k}^{+\infty} A_{j} \supseteq A_{m}
$$

Therefore, by monotone convergence,

$$
\left|A_{\star}\right|=\lim _{m \nearrow \infty}\left|\bigcap_{k=0}^{m} \bigcup_{j=k}^{+\infty} A_{j}\right| \geqslant \lim _{m \nearrow \infty}\left|A_{m}\right| \geqslant(1-\mu)\left|B_{1}\right| .
$$

Let $D \subset B_{1}$ denote the set of differentiability points of $u$ (recall that $D$ has full measure). We claim that

$$
\sup _{x \in A_{\star} \cap D}\left|\nabla u(x)-M e_{1}\right|<\mu^{1 / 4} .
$$

For this, we take $x \in A_{\star} \cap D$. By definition of $A_{\star}$ there exists a subsequence $j_{k} \nearrow \infty$ such that $x \in A_{j_{k}}$, thus, by (2.3),

$$
u\left(x+t_{j_{k}} e_{1}\right)-u(x) \geqslant(M-\mu) t_{j_{k}} .
$$

Dividing by $t_{j_{k}}>0$ and letting $k \nearrow \infty$ we obtain (recall that $t_{j_{k}} \searrow 0$ as $k \nearrow \infty$ )

$$
\partial_{1} u(x) \geqslant(M-\mu) \text {. }
$$

As a consequence

which gives

$$
M^{2} \geqslant|\nabla u(x)|^{2} \geqslant(M-\mu)^{2}+\sum_{i=2}^{n}\left|\partial_{i} u(x)\right|^{2},
$$

$$
\sum_{i=2}^{n}\left|\partial_{i} u(x)\right|^{2} \leqslant 2 M \mu
$$


Also (2.6) and the fact that $\partial_{1} u(x) \leqslant M$ imply that

$$
-\mu \leqslant \partial_{1} u(x)-M \leqslant 0
$$

hence $\left|\partial_{1} u(x)-M\right| \leqslant \mu$. We conclude that

$$
\left|\nabla u(x)-M e_{1}\right|^{2}=\left|\partial_{1} u(x)-M\right|^{2}+\sum_{i=2}^{n}\left|\partial_{i} u(x)\right|^{2} \leqslant \mu^{2}+2 M \mu<\sqrt{\mu}
$$

provided $\mu$ is sufficiently small, proving (2.5).

By (2.4) and (2.5) we deduce that

$$
\left|\left\{x \in B_{1}:\left|\nabla u(x)-M e_{1}\right| \geqslant \mu^{1 / 4}\right\}\right| \leqslant\left|B_{1} \backslash A_{\star}\right| \leqslant \mu\left|B_{1}\right| \leqslant \mu^{1 / 4} .
$$

Hence, if $\mu$ is small enough, we can apply Lemma 2.1 with $\delta=\mu^{1 / 4}$ to obtain the desired result.

\section{A REMARK ON FLAT BLOW-DOWNS}

First of all, we recall here the notion of blow-up and blow-down of a $s$-minimal surface $E$, which will be used in the proofs of Theorems 1.1 and 1.2

Assume that $0 \in \partial E$, define the family of sets $E_{r}:=E / r$, and let $E_{0}$ (resp. $E_{\infty}$ ) be a cluster point with respect to the $L_{\text {loc }}^{1}$-convergence for $E_{r}$ as $r \searrow 0$ (resp. $r \nearrow \infty$ ).

With this notation, $E_{0}$ is called a "blow-up" of $E$ (at 0 ), while $E_{\infty}$ is called a "blow-down". By [3, Theorem 9.2] we know that both $E_{0}$ and $E_{\infty}$ are $s$-minimal cones passing through the origin.

In the proof of Theorem 1.2 we will use the following observation:

Lemma 3.1. If $E_{\infty}$ is a half-space, then $E=E_{\infty}$.

Proof. Up to a rotation we can assume that $E_{\infty}=\left\{(x, \tau) \in \mathbb{R}^{n} \times \mathbb{R}: \tau \leqslant 0\right\}$. Let $r_{k} \nearrow \infty$ be a sequence such that $E_{r_{k}} \rightarrow E_{\infty}$, and let $\varepsilon_{0}$ be the universal flatness parameter in [3, Theorem 6.1]. By the uniform density estimates for $s$-minimal surfaces (see [3, Theorem 4.1]), we have that $\left(\partial E_{r_{k}}\right) \cap B_{1} \rightarrow\left(\partial E_{\infty}\right) \cap B_{1}$ in the Hausdorff distance as $r_{k} \nearrow \infty$. Hence, for $r_{k}$ sufficiently large $E_{r_{k}} \cap B_{1}$ lies in an $\varepsilon_{0}$-neighborhood of $E_{\infty}$, and [3, Theorem 6.1] yields that $\left(\partial E_{r_{k}}\right) \cap B_{1 / 2}$ is a $C^{1, \alpha}$-graph parameterized by a function $u_{r_{k}}: B_{1} \rightarrow \mathbb{R}$, with $\left\|u_{r_{k}}\right\|_{C^{1, \alpha}\left(B_{1 / 2}\right)} \leqslant C$ for some universal constants $\alpha \in(0,1)$ and $C>0$.

Scaling back, we deduce that $(\partial E) \cap B_{r_{k} / 2}$ coincides with the graph of a function $u$ which satisfies $u(x)=r_{k} u_{r_{k}}\left(x / r_{k}\right)$ and $u(0)=0$ (since $0 \in \partial E$ ). Hence

$$
r_{k}^{\alpha}[\nabla u]_{C^{\alpha}\left(B_{r_{k} / 2}\right)}=\left[\nabla u_{r_{k}}\right]_{C^{\alpha}\left(B_{1 / 2}\right)} \leqslant C
$$

and by letting $r_{k} \nearrow \infty$ we see that $\nabla u$ is constant. Thus $u$ is a linear function, which implies that $E$ is a half-space. Since $0 \in \partial E$ it is immediate to check that $E=E_{r}$ for all $r>0$, therefore (by letting $r \nearrow \infty$ ) $E=E_{\infty}$ as desired.

\section{Proof of Theorem 1.1}

By [3, Theorem 6.1] and [2, Theorem 5], there exists $\varepsilon_{0}>0$ such that, if $B_{r}(X) \subset B_{1}$ and $(\partial E) \cap B_{r}(X)$ lies in a slab of height $2 \varepsilon_{0} r$, then $(\partial E) \cap B_{r / 2}(X)$ is $C^{\infty}$. Hence we only need to show that, for any $X \in B_{1}$, there exists a radius $r<1-|X|$ such that $(\partial E) \cap B_{r}(X)$ lies in a slab of height $2 \varepsilon_{0} r$.

So, we fix $X_{0} \in B_{1}$, we suppose (up to a change of coordinates) that $\partial E$ is a Lipschitz graph in the $e_{n+1}$-direction in a neighborhood of $X$, and we assume by contradiction that, for any $r>0$ small, $(\partial E) \cap B_{r}\left(X_{0}\right)$ is never trapped inside a slab of height $2 \varepsilon_{0} r$. 
Up to translate the system of coordinate we can assume that $X_{0}=0$, and we consider a blow-up $E_{0}$ of $E$ (recall the notation of blow-ups presented in Section $\underline{3}$ ). By [3, Theorem 9.2] we know that $E_{0}$ is a Lipschitz $s$-minimal cone passing through the origin, and, by uniform density estimates for $s$-minimal surfaces (see [3, Theorem 4.1]), it is immediate to check that $\left(\partial E_{0}\right) \cap B_{R}$ is never trapped inside a slab of height $2 \varepsilon_{0} R$ for any $R>0$.

Now, up to a standard "dimension reduction argument" (see [3, Theorem 10.3]) we can "remove" all the singular points of $\partial E_{0}$ except the origin and we end upl with the following situation: $E_{0}$ is a Lipschitz cone passing through the origin,

$$
\left(\partial E_{0}\right) \cap B_{1} \text { does not lie in a slab of height } \varepsilon_{0},
$$

and $\partial E_{0}$ is a Lipschitz graph in the $e_{n+1}$-direction which is smooth outside the origin, that is

$$
\begin{gathered}
E_{0}=\left\{X=(x, \tau) \in \mathbb{R}^{n} \times \mathbb{R}: \tau<u(x)\right\}, \\
u(0)=0, \quad u \in C^{2}\left(\mathbb{R}^{n} \backslash\{0\}\right), \quad|\nabla u(x)| \leqslant M \quad \text { for any } x \neq 0 .
\end{gathered}
$$

To be precise, the dimension reduction argument in [3] gives that $u \in C^{1, \alpha}\left(\mathbb{R}^{n} \backslash\{0\}\right)$, and by $\left[2\right.$, Theorem 1] we obtain that $u \in C^{\infty}\left(\mathbb{R}^{n} \backslash\{0\}\right)$. Of course we can take $M>0$ to be the smallest possible (i.e., $M$ is the optimal Lipschitz constant of $u$ ).

Take $\mu_{0}:=\mu\left(n, \varepsilon_{0} / 2, M\right)$ as in Proposition 2.2. Then it follows from (4.7) that (2.2) cannot hold true. Hence, for any $\sigma \in \partial B_{1}$ there exists $t_{\sigma}>0$ such that

$$
\left|\left\{x \in B_{1}: u(x+t \sigma)-u(x)<\left(M-\mu_{0}\right) t\right\}\right| \geqslant \mu_{0}\left|B_{1}\right|
$$

for all $t \in\left(0, t_{\sigma}\right)$. Now we take $w_{0} \in C^{\infty}(\mathbb{R},[0,1])$, with $w_{0}(t)=0$ for any $t \in(-\infty, 1 / 4] \cup$ $[3 / 4,+\infty)$ and $w_{0}(t)=1$ for any $t \in[2 / 5,3 / 5]$. We set $w(x)=w_{0}(|x|)$ and we observe that

$$
w(x)=1 \text { for any } x \in B_{3 / 5} \backslash B_{2 / 5} .
$$

Our goal is to show that there exists a constant $\vartheta>0$ such that

$$
u(x+t \sigma) \leqslant u(x)+M t-\vartheta t w(x) \quad \forall x \in B_{1}, t \in\left(0, t_{\sigma}\right), \sigma \in \partial B_{1} .
$$

Before proving (4.11) we observe that, once (4.11) is established, we easily reach a contradiction and complete the proof of Theorem 1.1. Indeed, letting $t \searrow 0$ in (4.11) and using (4.10) we deduce that

$$
\nabla u(x) \cdot \sigma \leqslant M-\vartheta w(x)=M-\vartheta \quad \forall x \in B_{3 / 5} \backslash B_{2 / 5}, \sigma \in \partial B_{1},
$$

hence

$$
|\nabla u(x)| \leqslant M-\vartheta \quad \forall x \in B_{3 / 5} \backslash B_{2 / 5}
$$

by the arbitrariness of $\sigma$. Since $\nabla u$ is homogeneous of degree zero it follows that $|\nabla u(x)| \leqslant M-\vartheta$ for any $x \neq 0$, which contradicts our assumption that $M$ was the optimal Lipschitz constant of $u$. So, it only remains to prove (4.11).

For this we consider the surfaces

$$
\begin{array}{ll} 
& F:=\left\{(x, \tau) \in \mathbb{R}^{n} \times \mathbb{R}: \tau<u(x+t \sigma)\right\} \\
\text { and } \quad & G_{\vartheta, \alpha}:=\left\{(x, \tau) \in \mathbb{R}^{n} \times \mathbb{R}: \tau<u(x)+(M-\vartheta w(x)) t+\alpha\right\} .
\end{array}
$$

Notice that (4.11) is equivalent to

$$
F \subseteq G_{\vartheta, 0}
$$

\footnotetext{
${ }^{1}$ We notice that, since $\partial E_{0}$ is a Lipschitz graph, one can perform the dimension reduction argument without changing system of coordinates. Therefore, after a finite number of blow-ups, we still end up with a Lipschitz graph.
} 
To prove (4.12) we first observe that

$$
u(x+t \sigma) \leqslant u(x)+M t \leqslant u(x)+(M-\vartheta w(x)) t+M t
$$

provided $\vartheta \leqslant M$, thus $F \subseteq G_{\vartheta, \alpha}$ for any $\alpha>M t$. Now we reduce $\alpha$ till we find a critical $\alpha_{0}$ for which $G_{\vartheta, \alpha_{0}}$ touches $F$ from above. We claim that

$$
\alpha_{0} \leqslant 0 \text {. }
$$

Suppose by contradiction that $\alpha_{0}>0$. Since $u$ is $M$-Lipschitz we have that

$$
u(x+t \sigma) \leqslant u(x)+M t<u(x)+M t+\alpha_{0} .
$$

which implies that $G_{\vartheta, \alpha_{0}}$ and $F$ can only touch at a some point $X_{0}=\left(x_{0}, t_{0}\right) \in \mathbb{R}^{n} \times \mathbb{R}$ with $x_{0} \in \operatorname{supp}(w) \subset B_{3 / 4} \backslash B_{1 / 4}$. Hence, it is easy to see (by compactness) that a contact point $X_{0}=\left(x_{0}, t_{0}\right)$ exists, and since $x_{0} \in B_{3 / 4} \backslash B_{1 / 4}$ we have that both sets are uniformly $C^{2}$ near $X_{0}$, so the $s$-mean curvature operators $I[F]$ and $I\left[G_{\vartheta, \alpha_{0}}\right]$ (recall (1.1)) may be computed at $X_{0}$ in the classical sense.

Since $F$ and $G_{0, \alpha_{0}}$ are $s$-minimal surfaces, we have that

$$
I[F]\left(X_{0}\right)=0=I\left[G_{0, \alpha_{0}}\right]\left(X_{0}\right) .
$$

Also, since $G_{\vartheta, \alpha_{0}}$ is a $C^{2}$-diffeomorphism of $G_{0, \alpha_{0}}$ of size $\vartheta t$, and $G_{\vartheta, \alpha_{0}}$ is uniformly $C^{2}$ in a neighborhood of $X_{0}$, we have that

$$
\left|I\left[G_{\vartheta, \alpha_{0}}\right]\left(X_{0}\right)\right| \leqslant C \vartheta t
$$

for some universal constant $C>0$. Furthermore, since $F \subseteq G_{\vartheta, \alpha_{0}}$ we have that

$$
\chi_{G_{\vartheta, \alpha_{0}}}-\chi_{G_{\vartheta, \alpha_{0}}^{c}}-\chi_{F}+\chi_{F^{c}}=2 \chi_{G_{\vartheta, \alpha_{0}} \backslash F} .
$$

Now we define

$$
Z:=\left\{x \in B_{1}: u(x+t \sigma)-u(x)<\left(M-\mu_{0}\right) t\right\} \subset \mathbb{R}^{n}
$$

and

$$
W:=\left\{(x, \tau) \in \mathbb{R}^{n} \times \mathbb{R}: x \in Z \text { and } u(x+t \sigma)<\tau<u(x+t \sigma)+\mu_{0} t / 2\right\} \subset \mathbb{R}^{n+1} .
$$

We remark that $|Z| \geqslant \mu_{0}\left|B_{1}\right|$ thanks to (4.9), therefore $|W| \geqslant \mu_{0}^{2} t\left|B_{1}\right| / 2$. (Notice that, by abuse of notation, we are using $|\cdot|$ to denote both the Lebesgue measure in $\mathbb{R}^{n}$ and $\mathbb{R}^{n+1}$.)

We claim that

$$
\left(G_{\vartheta, \alpha_{0}} \backslash F\right) \supseteq W
$$

provided $\vartheta$ is sufficiently small. Indeed, let $(x, \tau) \in W$. Then $x \in Z$ and $u(x+t \sigma)<\tau<$ $u(x+t \sigma)+\mu_{0} t / 2$. This says that $(x, \tau) \notin F$ and

$$
\begin{gathered}
\tau<u(x+t \sigma)-\frac{\mu_{0} t}{2}<u(x)+\left(M-\mu_{0}\right) t+\frac{\mu_{0} t}{2} \\
\leqslant u(x)+M t-\vartheta t \leqslant u(x)+M t-\vartheta w(x) t
\end{gathered}
$$

provided $\vartheta \in\left(0, \mu_{0} / 4\right)$. This shows that $(x, \tau) \in G_{\vartheta, \alpha_{0}}$ proving (4.17).

Since by construction $Z \subseteq B_{1} \subset \mathbb{R}^{n}$ and $u$ is $M$-Lipschitz with $u(0)=0$, we deduce that $W \subset$ $B_{1} \times[-1-2 M, 1+2 M]$, which implies that

$$
\sup _{Y \in W}\left|X_{0}-Y\right| \leqslant C_{M}
$$

for some $C_{M}>0$, and (by (4.17)) that

$$
\left|\left(G_{\vartheta, \alpha_{0}} \backslash F\right) \cap W\right|=|W| \geqslant \frac{\mu_{0}^{2} t\left|B_{1}\right|}{2} .
$$


From this, (1.1), and (4.16), we conclude that

$$
\begin{aligned}
I\left[G_{\vartheta, \alpha_{0}}\right]\left(X_{0}\right)-I[F]\left(X_{0}\right) & =\int_{\mathbb{R}^{n+1}} \frac{2 \chi_{G_{\vartheta, \alpha_{0}} \backslash F}(Y)}{\left|X_{0}-Y\right|^{n+1+s}} d y \\
& \geqslant \int_{W} \frac{2 \chi_{G_{\vartheta, \alpha_{0}} \backslash F}(Y)}{\left|X_{0}-Y\right|^{n+1+s}} d y \\
& \geqslant 2 C_{M}^{-n-1-s} \int_{W} \chi_{G_{\vartheta, \alpha_{0}} \backslash F}(Y) d y \\
& =2 C_{M}^{-n-1-s}|W| \\
& \geqslant C_{M}^{-n-1-s} \mu_{0}^{2} t\left|B_{1}\right| .
\end{aligned}
$$

Hence, combining (4.14) and (4.15) we get

$$
C \vartheta t \geqslant I\left[G_{\vartheta, \alpha_{0}}\right]\left(X_{0}\right)=I\left[G_{\vartheta, \alpha_{0}}\right]\left(X_{0}\right)-I[F]\left(X_{0}\right) \geqslant C_{M}^{-n-1-s} \mu_{0}^{2} t\left|B_{1}\right|,
$$

which is a contradiction if $\vartheta$ is sufficiently small. This contradiction proves (4.13), that in turn implies (4.12) and so (4.11). This concludes the proof of Theorem 1.1.

\section{Proof of Theorem 1.2}

Let $E_{\infty}$ be a blow-down of $E$, that is a cluster point for $E_{r}:=E / r$ as $r \nearrow \infty$. In this way we get a $s$-minimal cone, and the assumption that no singular $s$-minimal cones exist in dimension $n$ combined with a standard dimension reduction argument implies that $E_{\infty}$ can only be singular at the origin.

Also, because $\partial E$ was a graph, $E_{\infty}$ is an hypograph in $\mathbb{R}^{n+1}$, that is

$$
(x, \tau) \in E_{\infty} \quad \Longrightarrow \quad(x, \tau-t) \in E_{\infty} \quad \forall t \geqslant 0 .
$$

Now we show that $E_{\infty}$ is in fact a graph (and not only an hypograph). For this, suppose by contradiction that there exists $\tau_{\infty}>0$ such that $\partial E_{\infty}$ touches $\partial E_{\infty}+\tau_{\infty} e_{n+1}$ at some point. Then, by the strong maximum principle 2 we get $E_{\infty}=E_{\infty}+\tau_{\infty} e_{n+1}$, from which (iterating this equality) we get $E_{\infty}=E_{\infty}+k \tau_{\infty} e_{n+1}$ for any $k \in \mathbb{N}$. This fact combined with (5.19) implies that

$$
E_{\infty}=\mathscr{C} \times \mathbb{R}
$$

where $\mathscr{C}$ is a $s$-minimal cone in $\mathbb{R}^{n}$. Hence it follows by our assumption that $\mathscr{C}$ is a half-space, and Lemma 3.1 gives that $E=\mathscr{C} \times \mathbb{R}$ which is in contradiction with the fact that $E$ was a graph.

Hence we have shown that $\partial E_{\infty}$ and $\partial E_{\infty}+\tau e_{n+1}$ never touch for any $\tau>0$, which implies that $\partial E_{\infty}$ is the graph of a function $u_{\infty}: \mathbb{R}^{n} \rightarrow \mathbb{R}$. In addition, since $E_{\infty}$ is smooth outside the origin, so is $u_{\infty}$.

Now, as in the proof of Theorem 1.1, we consider a bump function $w_{0} \in C^{\infty}(\mathbb{R},[0,1])$, with $w_{0}(t)=0$ for any $t \in(-\infty, 1 / 4] \cup[3 / 4,+\infty)$ and $w_{0}(t)=1$ for any $t \in[2 / 5,3 / 5]$, and we define $w(x)=w_{0}(|x|)$. Then, we fix $\sigma \in \partial B_{1}$ and consider the family of sets

$$
F_{t}:=\left\{(x, \tau): \tau \leqslant u_{\infty}(x+t \vartheta w(x) \sigma)-t\right\},
$$

where $t \in[0,1]$ and $\vartheta>0$. By compactness we see that, if $\vartheta$ is sufficiently small, then $F_{1} \subseteq E_{\infty}$. Let $t_{0} \in[0,1]$ be the smallest $t$ for which $F_{t} \subseteq E_{\infty}$, and assume by contradiction that $t_{0}>0$.

\footnotetext{
${ }^{2} \mathrm{~A}$ simple and direct way to see the strong maximum principle is to use that $E_{\infty}$ and $E_{\infty}+\tau_{\infty} e_{n+1}$ are smooth cones outside the origin. So, if they touch, we can find a contact point $X_{0} \neq 0$, and by computing the operator $I$ defined in (1.1) at $X_{0}$ for both surfaces, since both $E_{\infty}$ and $E_{\infty}+\tau_{\infty} e_{n+1}$ are $s$-minimal and $E_{\infty} \subset E_{\infty}+\tau_{\infty} e_{n+1}$ we get

$$
0=I\left[E_{\infty}+\tau_{\infty} e_{n+1}\right]\left(X_{0}\right)-I\left[E_{\infty}\right]\left(X_{0}\right)=\int_{\mathbb{R}^{n+1}} \frac{2 \chi_{E_{\infty}+\tau_{\infty} e_{n+1} \backslash E_{\infty}}(Y)}{\left|X_{0}-Y\right|^{n+1+s}} d y,
$$

which implies that $E_{\infty}=E_{\infty}+\tau_{\infty} e_{n+1}$, as desired.
} 
Since $E_{\infty}$ is a graph, we see that $F_{t_{0}}$ can only touch $E_{\infty}$ from below at some point $X_{0}=\left(x_{0}, t_{0}\right)$ with $x_{0} \in \operatorname{supp}(w) \subset B_{3 / 4} \backslash B_{1 / 4}$. Hence, it is easy to see (by compactness) that a contact point $X_{0}=\left(x_{0}, t_{0}\right)$ exists, and since $x_{0} \in B_{3 / 4} \backslash B_{1 / 4}$ we have that both sets are smooth near $X_{0}$.

Therefore we can easily adapt the arguments provided in (4.14)-(4.18) as follows: First, by the $s$-minimality of $E_{\infty}$ we have that $I\left[E_{\infty}\right]\left(X_{0}\right)=0=I\left[F_{0}\right]\left(X_{0}\right)$. Also, since $F_{t_{0}}$ is a $C^{2}$ diffeomorphism of $F_{0}$ of size $\vartheta t_{0}$ and $F_{0}$ is uniformly $C^{2}$ in a neighborhood of $X_{0}$, we have that

$$
\left|I\left[F_{t_{0}}\right]\left(X_{0}\right)\right| \leqslant C \vartheta t_{0} .
$$

On the other hand, since the graph of $u_{\infty}$ is uniformly Lipschitz in a non-trivial fraction of points (just pick a point where the tangent space to $\partial E_{\infty}$ is not vertical and consider a small neighborhood of this point) we see that $\partial F_{t_{0}}$ and $\partial E_{\infty}$ lie at distance $\geqslant c t_{0}$ on a non-trivial fraction of points, therefore

$$
\left|\left(E_{\infty} \backslash F_{t_{0}}\right) \cap B_{1}\right| \geqslant c_{0} t_{0}
$$

for some $c_{0}>0$. Hence, arguing as in the proof of Theorem 1.1 we get

$$
\left|I\left[F_{t_{0}}\right]\left(X_{0}\right)\right|=\left|I\left[F_{t_{0}}\right]\left(X_{0}\right)-I\left[E_{\infty}\right]\left(X_{0}\right)\right| \geqslant c^{\prime} t_{0}
$$

for some $c^{\prime}>0$, which is in contradiction with (5.20) if $\vartheta$ was chosen sufficiently small.

This proves that $t_{0}=0$, which implies that $F_{t} \subseteq E_{\infty}$ for any $t \in(0,1)$, or equivalently

$$
\frac{u_{\infty}(x+t \vartheta w(x) \sigma)-u_{\infty}(x)}{t} \leqslant 1 \quad \forall t \in(0,1) .
$$

Hence, letting $t \searrow 0$ we obtain

$$
\vartheta w(x) \nabla u_{\infty}(x) \cdot \sigma \leqslant 1 \quad \forall x \in \mathbb{R}^{n} \backslash\{0\}, \sigma \in \partial B_{1},
$$

which combined with the fact that $w=1$ in $B_{3 / 5} \backslash B_{2 / 5}$ and $\sigma \in \partial B_{1}$ is arbitrary implies

$$
\left|\nabla u_{\infty}(x)\right| \leqslant 1 / \vartheta \quad \forall x \in B_{3 / 5} \backslash B_{2 / 5} .
$$

Since $u_{\infty}$ is 1 -homogeneous we deduce that $u_{\infty}$ is globally Lipschitz. So by Theorem 1.1 it is smooth also at the origin, hence (being a cone) $E_{\infty}$ a half-space. Using again Lemma 3.1 we deduce that $E$ is a half-space as well, concluding the proof of Theorem 1.2 ,

\section{REFERENCES}

[1] L. Ambrosio, G. De Philippis, and L. Martinazzi, Gamma-convergence of nonlocal perimeter functionals, Manuscripta Math. 134 (2011), no. 3-4, 377-403, DOI 10.1007/s00229-010-0399-4. MR2765717 (2012d:49084)

[2] B. Barrios, A. Figalli, and E. Valdinoci, Bootstrap regularity for integro-differential operators and its application to nonlocal minimal surfaces, Ann. Sc. Norm. Super. Pisa Cl. Sci. (5).

[3] L. Caffarelli, J.-M. Roquejoffre, and O. Savin, Nonlocal minimal surfaces, Comm. Pure Appl. Math. 63 (2010), no. 9, 1111-1144, DOI 10.1002/cpa.20331. MR2675483 (2011h:49057)

[4] L. Caffarelli and E. Valdinoci, Uniform estimates and limiting arguments for nonlocal minimal surfaces, Calc. Var. Partial Differential Equations 41 (2011), no. 1-2, 203-240, DOI 10.1007/s00526-010-0359-6. MR2782803 (2012i:49064)

[5] E. De Giorgi, Una estensione del teorema di Bernstein, Ann. Scuola Norm. Sup. Pisa (3) 19 (1965), 79-85.

[6] O. Savin and E. Valdinoci, Regularity of nonlocal minimal cones in dimension 2, Calc. Var. Partial Differential Equations, DOI 10.1007/s00526-012-0539-7, (to appear in print).

The University of Texas at Austin, Mathematics Department RLM 8.100, 2515 Speedway Stop C1200, Austin TX 78712-1202 (USA)

E-mail address: figalli@math.utexas.edu

Weierstrass Institut für Angewandte Analysis und Stochastik, Mohrenstrasse 39, D-10117 Berlin (GERMANY)

E-mail address: enrico.valdinoci@wias-berlin.de 